\title{
Efectos de la colaboración de la industria del mueble de España con los proveedores de servicios
}

DOI: 10.22403/UQROOMX/TYP06/03

\section{Whe}

Resumen

\author{
Gonzalo Maldonado Guzmán* \\ María del Carmen Martínez Serna** \\ Luis Aguilera Enríquez*** \\ Universidad Autónoma de Aguascalientes
}

Este trabajo tiene como objetivo principal analizar los efectos que la colaboración con los proveedores de servicios ejerce en el desempeño de las empresas que integran la industria del mueble en España. El análisis empírico se llevó a cabo con una muestra de 322 empresas productoras de muebles con 20 o más trabajadores, y que al menos hubieran realizado una exportación o participado en la Feria Internacional del Mueble de Valencia en las ediciones 2005 o 2006.

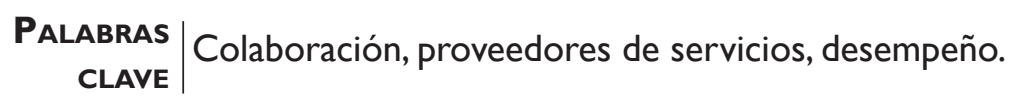

*correo electrónico: gmaldona@correo.uaa.mx

**correo electrónico: mcmartin@correo.uaa.mx

****correo electrónico: laguiler@correo.uaa.mx 


\section{Introducción}

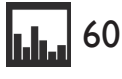

El nuevo milenio se caracteriza por un constante desarrollo de la información y de la globalización económica, lo que ha llevado a las empresas a plantearse nuevas estrategias de participación en los mercados. Una de ellas es la colaboración, que en la actualidad es aplicada en un importante número de organizaciones empresariales para obtener una ventaja competitiva (Dyer y Singh, 1998; Simatupang y Sridharan, 2005a; Stefansson, 2006). Participar en un ambiente de colaboración permite que todas las empresas involucradas se orienten a la obtención de beneficios mutuos, satisfaciendo la demanda del mercado con los suministros requeridos (Fisher, 1997). En este sentido, lograr beneficios para todos los miembros de la cadena de suministro posibilita un retorno positivo de las inversiones y la eficiencia en la gestión de los inventarios (Walker, Bovet y Martha, 2000; Simatupang y Sridharan, 2005b; Wilding y Humphries, 2006).

Una de las ventajas más significativas de la colaboración con los proveedores en la cadena de suministro es que en el interior de las organizaciones se entiende la necesidad de alinear los esfuerzos de las empresas participantes para mejorar el desempeño tanto de la colaboración en sí como de las organizaciones (Lambert, Knemeyer y Gardner, 2004; Simatupang y Sridharan, 2005b; Thron, Nagy y Wassan, 2006). Sin embargo, a pesar de la publicación de un gran número de trabajos sobre el tema, algunas investigaciones sostienen que la colaboración es un fenómeno unilateral que se dirige sólo a un rasgo específico del proceso, por ejemplo, una parte de la información de la cadena de suministro o una parte de la gestión de los inventarios (Lee, Padmanabhan y Whang, 1997; Simatupang y Sridharan, 2005b; Stefansson, 2006; Thron, Nagy y Wassan, 2006; Wilding y Humphries, 2006;Adobor, 2006).Adicionalmente, estudios recientes han puesto especial atención en el análisis de los rasgos de cada faceta de la colaboración. Lo anterior ayuda a entender, en parte, por qué las organizaciones que intervienen en el proceso de la colaboración encuentran serias dificultades en la comprensión del término y en los resultados obtenidos (Mentzer, Foggin y Golicic, 2000; Stefansson, 2006;Wilding y Humphries, 2006). En la actualidad, un buen número de las investigaciones se ha dedicado a la definición de la colaboración en las actividades de la cadena de suministro, porque entre los rasgos de la colaboración descubren claramente la interacción del fenómeno (Simatupang,Wright y Sridharan, 2002; Simatupang y Sridharan, 2002 y 2005a; Thron, Nagy y Wassan, 2006). 


\section{Revisión de la literatura}

La era de la información y la globalización están conduciendo a las organizaciones empresariales a utilizar la colaboración como una estrategia novedosa y un recurso para lograr una mayor ventaja competitiva (Dyer y Singh, 1998; Simatupang y Sridharan, 2005a). Implantar un trabajo conjunto permite que todos los participantes en la colaboración generen y obtengan beneficios y ventajas mutuos, igualando la demanda con el suministro (Fisher, 1997; Simatupang y Sridharan, 2005a), lo cual a menudo representa un retorno positivo de la inversión y una gestión más eficiente de los inventarios (Walker, Bovet y Martha, 2000).

El término colaboración, que literalmente significa "trabajo conjunto" (Huxham, 1996; Jordan y Michel, 2000; Bititci et al., 2004; Stefansson, 2006), ha sido conceptuado desde distintas perspectivas por investigadores y académicos. No obstante, la mayoría de los que se dedican al estudio de este tema han asumido que la colaboración sólo se analiza desde un punto de vista, pues suele aplicarse únicamente a una parte del proceso y no a la colaboración en su conjunto. Por ejemplo, los integrantes de la cadena de suministro se reúnen para examinar y rediseñar una parte del proceso de la colaboración, la cual resulta ser la más apropiada para mejorar el desempeño de las organizaciones empresariales involucradas, y no la colaboración en su totalidad (Corbett, Blackburn y Van Wassenhove, 1999; Lambert y Cooper, 2000; Simatupang y Sridharan, 2005a). Adicionalmente, la colaboración ha sido analizada como una nueva forma de conseguir una ventaja competitiva en los negocios, sobre todo en aquellas situaciones en las cuales las empresas por sí solas no pueden alcanzar las metas trazadas (Huxham, 1996).

El desarrollo de la colaboración tiene como premisa que una empresa no puede prosperar compitiendo por sí sola. Por ello, hoy en día una gran cantidad de negocios aplican un proceso de colaboración con otras organizaciones empresariales, logrando trabajar colaborativamente en el largo plazo y aspirando a obtener mejores resultados (Stern y Reeve, 1980;Anderson y Narus, 1990; Simatupang y Sridharan, 2005a; Min et al., 2005; Stefansson, 2006; Vereecke y Muylle, 2006). Pero, en la misma medida, las empresas que trabajan en un proceso de colaboración en una o varias actividades en particular comparten riesgos y beneficios (Lambert, Emmelhainz y Gardner, 1999; Stefansson, 2006). 
A pesar de lo redituable de la colaboración para las organizaciones participantes, Sabath y Fontanella (2002) aseguran que ésta es la mayor desilusión que se ha tenido de las distintas estrategias de gestión de la cadena de suministro introducidas hoy en día. Para contrarrestar las críticas de estos inves-tigadores, la colaboración ha sido analizada con amplitud por disciplinas como la sociología (Powell et al., 2005), la psicología (Stern y Hicks, 2000; Konczak, 200 I), mercadotecnia (Jap, 1999; Perks, 200 I; Gadde, Humer y Hakansson, 2003), la gestión (Sawhney, 2002; Cross, Borgatti y Parker, 2002; Singh y Mitchell, 1996) y la gestión de la cadena de suministro (Tuominen, 2004; Min et al., 2005; Holweg et al., 2005; Simatupang y Sridharan, 2005a; Stefansson, 2006;Wilding y Humphries, 2006; Thron, Nagy y Wassan, 2006; Vereecke y Muylle, 2006).

En la literatura actual sobre la colaboración se pueden identificar dos enfoques: el que la entiende como un proceso de interorganización de las empresas, y el que la considera una base de las relaciones interorganizacionales de las empresas. En el primero, la colaboración es vista como un proceso de gestión de los negocios en el cual las organizaciones participantes trabajan de manera conjunta para obtener los beneficios y las metas planteados (Stank, Keller y Daugherty, 200 I; Mentzer et al., 200 I; Min et al., 2005;Adobor, 2006; Stefansson, 2006; Vereecke y Muylle, 2006); además incluye participar de manera conjunta en la toma de decisiones (Stank, Keller y Daugherty, 200 I) y en la solución de problemas (Spekman, Salmond y Lambe, 1997), así como compartir información de la cadena de suministro (Stank, Daugherty y Autry, 1999; Sabath y Fontanella, 2002; Thron, Nagy y Wassan, 2006;Vereecke y Muylle, 2006).

En cambio, el segundo enfoque concibe la colaboración como una formación o unión de diversas organizaciones empresariales en donde todos los miembros trabajan colectivamente compartiendo información y recursos, lo que conlleva disminuir riesgos, pues todos procuran cumplir con los mismos objetivos (Sriram, Krapfel y Spekman, 1992; Ellram y Edis, 1996; Bowersox, Closs y Stank, 2003; Golicic, Foggin y Mentzer, 2003; Min et al., 2005). Para dar cumplimiento a los compromisos generados por la colaboración, ésta tiene que desarrollarse en el interior de las áreas funcionales de cada organización empresarial (Vereecke y Muylle, 2006). Esta función interna es interdependiente del proceso de colaboración entre los negocios y establece las bases para el correcto funcionamiento de la colaboración entre las diversas organizaciones. Como resultado, se produce una integración de las actividades internas y externas de las empresas. Así pues, las entidades participantes pueden ser 
funcionalmente interdependientes $y$, al mismo tiempo, buscar la obtención de beneficios para todos (Jap, 200 I; Min et al., 2005).

Un proceso de colaboración debe buscar la prosperidad de las organizaciones empresariales implicadas, lo cual significa realizar cambios sustanciales en el interior de los negocios, que faciliten el intercambio de información (Stank, Keller y Daugherty, 200 I; Simatupang y Sridharan, 2005a; Min et al., 2005; Vereecke y Muylle, 2006). Asimismo, un intercambio eficiente de información y la operación de planes conjuntos son necesarios para lograr los beneficios que brinda la colaboración (Quinn, 1999; Simatupang y Sridharan, 2005a; Min et al., 2005; Stefansson, 2006; Vereecke y Muylle, 2006).

En suma, la colaboración facilita el intercambio de información y la transferencia de conocimiento entre las organizaciones participantes (Simonin, 1997; Hardy, Phillips y Lawrence, 2003;Wilding y Humphries, 2006).

\section{Modelo teórico y planteamiento de hipótesis}

En este trabajo de investigación se intentará aportar alguna evidencia de los efectos que la colaboración con los proveedores tiene en el desempeño de la industria del mueble, pues se cree que repercute de manera positiva en el funcionamiento de las compañías de la industria del mueble de España. Una vez establecido este objetivo, se considera que la colaboración puede medirse desde tres perspectivas: innovación, reducción de los costos de las compras y desempeño financiero (véase figura I).

Varios estudios han demostrado que a menudo las relaciones son el recurso más importante en el desarrollo de nuevas ideas y de información (Von Hippel, 1988). La colaboración con los proveedores facilita el intercambio de conocimiento y aumenta la creación e innovación, que puede verterse sobre los proveedores (Inkpen, 1996), quienes cumplen una función trascendental en la exploración de los procesos de innovación (Walter et al., 2003). Por ello, la innovación es un elemento esencial para conseguir buenos resultados en las organizaciones empresariales, lo que permite a los negocios obtener una ventaja competitiva temporal que les genera un beneficio superior. Asimismo, la innovación es un prerrequisito de largo plazo para la supervivencia y el crecimiento de las compañías.

En consecuencia, en la mayoría de los negocios, la innovación es desarrollada colaborativamente por medio de una serie de empresas, sobre todo pequeñas y 


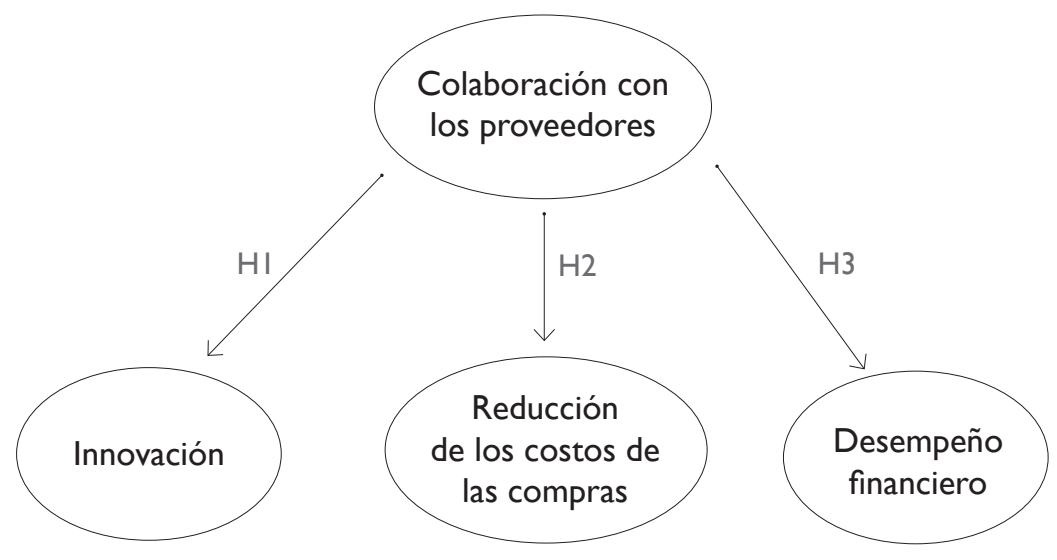

Figura I. Modelo teÓRICO

medianas (Jorde y Teece, 1990), que participan de manera conjunta (Powell, I998; Simatupang y Sridharan, 2005a). La colaboración con los proveedores es fundamental para la innovación (Powell, 1998), ya que las compras ejercen una fuerte influencia en el grado de innovación de las organizaciones empresariales. Los proveedores de servicios pueden contribuir en el desarrollo de la innovación en las empresas mediante sus propios recursos en I+D, absorbiendo algunos costos en los que, por lo general, tienen que incurrir las empresas (Powell, 1998; Corsten y Felde, 2005; Stefansson, 2006).

Hipótesis I (HI). Un alto nivel de colaboración con los proveedores genera un alto nivel de innovación en las empresas de la industria del mueble en España. La literatura actual sobre el tema establece que para las organizaciones empresariales que participan en un proceso de colaboración los costos de las compras incluyen, entre otros, los de levantamiento de pedidos y transporte (Williamson, 1985; Cannon y Homburg, 200 I; Corsten y Felde, 2005).Así pues, la colaboración reduce los costos de las compras gracias a una contracción de los costos, una comunicación frecuente, una mejor coordinación y una mayor unión para solucionar problemas operacionales (Scannell, Vickery y Dröge, 2000; Cannon y Homburg, 200 I; Corsten y Felde, 2005). Entonces, desde una perspectiva de los costos totales de los negocios, los costos de las compras determinan el desempeño de los proveedores de servicios (Ellram, 1995). 
En esta investigación se incluyeron los costos de comunicación, transporte y levantamiento de pedidos como una medida del desempeño.

Hipótesis $2(\mathrm{H} 2)$. Un elevado nivel de colaboración con los proveedores genera una reducción de los costos de las compras de las empresas de la industria del mueble en España. Sobre todo en sectores industriales tradicionales, como el del mueble, donde los proveedores de servicios frecuentemente ejercen una fuerte influencia en el desarrollo de los productos, en el retorno del capital y en las ventas, la colaboración ha ganado relevancia como una medida de desempeño (Dröge y Germain, 2000; Corsten y Felde, 2005); en este estudio se define al desempeño financiero como el retorno de capitales y de las ventas y el mejoramiento de las medidas para comparar los resultados de las industrias (Corsten y Felde, 2005).

Hipótesis $3(\mathrm{H} 3)$. Un elevado nivel de colaboración con los proveedores genera un alto desempeño de las finanzas de las empresas de la industria del mueble en España.

\section{Metodología}

Para validar las hipótesis propuestas se llevó a cabo una investigación empírica en las empresas de la industria del mueble en España. En concreto, se analizaron las relaciones de colaboración en el desempeño de las empresas productoras de muebles. La primera fase del estudio fue cualitativa, se efectuaron entrevistas en profundidad con los responsables de algunas empresas del ramo. Los resultados permitieron un mayor conocimiento de la situación del sector y fueron muy útiles para la correcta definición de la fase cualitativa.

Diseño de la muestra y recolección de los datos

Para determinar el marco de referencia de este trabajo se obtuvo el directorio de las empresas que contaban con 20 o más trabajadores, con el apoyo (hasta agosto de 2006) de la Asociación Nacional de Industriales y Exportadores de Muebles de España (ANIEME), que tenía 22I empresas asociadas. También se recurrió a la Feria Internacional del Mueble de Valencia (FIM) con el fin de conocer las fichas de las empresas españolas que participaron en las dos exposiciones previas al 
estudio (2005 y 2006) y seleccionar aquellas que tuvieran 20 o más empleados, obteniendo una relación de 279 empresas. De este modo se conformó un directorio final de 500 empresas, que representan poco más de $38 \%$ del total de la población objeto de estudio (I 300). Cabe señalar que las compañías asociadas a la ANIEME, al igual que las expositoras en la FIM, pertenecen a varias organizaciones empresariales, tanto regionales como nacionales, por lo cual la investigación no se centró en un grupo o asociación en particular.

De igual manera, la encuesta se diseñó para que fuera contestada por los gestores de las empresas de la industria del mueble de las 16 comunidades autónomas de España, y fue enviada por correo ordinario a cada una de las 500 empresas seleccionadas. Se obtuvo una tasa de respuesta de $53 \%$, pues se recibieron 334 contestaciones, 12 de las cuales se eliminaron por no reunir los requisitos establecidos, quedando un total de 322 encuestas validadas con un error de $4.8 \%$. Todos los entrevistados son profesionales en el campo del mueble, han trabajado en la industria durante varios años, e incluso algunos han estado en las empresas toda su vida activa. Esto permitió que los gerentes participantes proporcionaran información muy valiosa e interesante por el profundo conocimiento que tienen de la industria y del área que investigamos. El cuadro I resume los aspectos más relevantes del trabajo efectuado.

\section{Desarrollo de medidas}

Como paso previo al examen de los resultados, se llevó a cabo un análisis de fiabilidad y validez de las medidas utilizadas. Las cuatro variables empleadas -colaboración con los proveedores, innovación, reducción de los costos de las compras y desempeño financiero-, están definidas por escalas unidimensionales. Todos los ítems están construidos según una escala tipo Likert de cinco puntos, con "I = completamente en desacuerdo" y " 5 = completamente de acuerdo" como límites. La colaboración fue medida en una escala de cuatro ítems adaptada de Heide y John (1990); Zaheer, McEvily y Perrone, ( 1998); y Corsten y Felde (2005), cubriendo la unión de los procesos de desarrollo de productos. La escala para la innovación fue adaptada de Gilley y Rasheed (2000), y cubre el mejoramiento de los productos y la innovación de los procesos, así como una disminución en el gasto de investigación y desarrollo. La escala para la reducción de los costos de las compras fue tomada de Cannon y Homburg (200I), y comprende los costos de coordinación, entrega y transportación. 
Cuadro I. Ficha técnica de la inVestigación

\begin{tabular}{ll}
\hline Características & Encuesta \\
\hline Universo* & I 300 empresas de la industria del mueble \\
Ámbito de estudio & Nacional \\
Unidad muestral & $\begin{array}{l}\text { Empresas productoras de muebles con } 20 \\
\text { o más trabajadores }\end{array}$ \\
Método para obtener la información & Encuesta postal y entrevista personal \\
Procedimiento de muestreo & $\begin{array}{l}\text { Envío postal a todas las empresas } \\
\text { seleccionadas }\end{array}$ \\
Tamaño de la muestra & 500 empresas \\
Margen de error de muestreo & $+/-4 \%$ a un nivel global, para un nivel de \\
& $\begin{array}{l}\text { confianza de } 95 \%(\mathrm{P}=\mathrm{q}=0.5) \\
\text { Fecha del trabajo de campo }\end{array}$
\end{tabular}

*Fuente: Instituto Tecnológico del Mueble, Madera, Embalaje y Afines (AIDIMA).

Finalmente, la escala del desempeño financiero fue ajustada conforme criterios de Dröge y Germain (2000), y de Gilley y Rasheed (2000), la cual calcula, sólo para un período de tres años, el retorno de las ventas y el capital, así como un estado de las cuentas más general en la mejora de los resultados financieros.

\section{Fiabilidad y validez}

Para evaluar la fiabilidad y validez de las escalas de medida se realizó un análisis factorial confirmatorio (AFC), usando el método de máxima verosimilitud en EQS 6.I (Bentler, 2005; Brown, 2006; Byrne, 2006). Por su parte, la fiabilidad de las cuatro escalas de medida propuestas se evaluó a partir de los coeficientes $\alpha$ de Cronbach y el índice de fiabilidad compuesta (Bagozzi y Yi, 1988). Todos los valores de la escala excedieron el nivel recomendado de 0.7 para el $\alpha$ de Cronbach, que proporciona una evidencia de fiabilidad y justifica la fiabilidad interna de las escalas (Nunnally y Bernstein, 1994; Hair et al., 1995). Los datos analizados directamente de la base de datos demuestran una distribución no normal (estimación del coeficiente normalizado de Mardia $=40.20$ ). Asimismo, 
se emplearon otros métodos de estimación al asumir que la normalidad está presente. Para corregir los errores de los diferentes modelos de estimación utilizados seguimos las recomendaciones de Chou, Bentler y Satorra (1991), y las de Hu, Bentler y Kano (1992). De esta manera, los valores robustos (Satorra y Bentler, 1988) se usaron para proporcionar una mejor evidencia de los ajustes estadísticos.

Los ajustes que se manejaron en el modelo fueron el índice de ajuste normalizado (NFI), el índice de ajuste no normalizado (NNFI), el índice de ajuste comparativo (CFI) y la raíz cuadrada de la media del error de aproximación (RMSEA) (Bentler y Bonnet, 1980; Byrne, 1989; Bentler, 1990; Hair et al., 1995; Chau, 1997; Heck, 1998).Valores del NFI, NNFI y CFI entre 0.80 y 0.89 representan un ajuste razonable (Segars y Grover, 1993), y un valor igual o superior a 0.90 es prueba de un buen ajuste (Jöreskog y Sörbom, I 986; Byrne, 1989; Papke-Shields, Malhotra y Grover, 2002). Valores del RMSEA inferiores a 0.08 son aceptables (Jöreskog y Sörbom, 1986; Hair et al., 1995). Los resultados de la aplicación final del AFC se presentan en el cuadro 2 y sugieren que el modelo de medición final proporciona un buen ajuste de los datos con base en número de ajustes estadísticos $\left(S-B X^{2}=125.3174 ; d f=59 ; p=0.000 ; \mathrm{NFI}=0.972 ; \mathrm{NNFI}=0.980\right.$; $C F I=0.985 ; y$ RMSEA $=0.059$ ). Como evidencia de la validez convergente, los resultados del AFC indican que todos los ítems de los factores relacionados son significativos $(p<0.00 \mathrm{I})$, el tamaño de todas las cargas factoriales estandarizadas son superiores a 0.60 (Bagozzi y Yi, 1988) y el promedio de las cargas factoriales estandarizadas de cada factor superan sin problema alguno el valor de 0.70 (Hair et al., 1995).

El cuadro 2 muestra una alta consistencia interna de los constructos, en cada caso, el $\alpha$ de Cronbach excede el valor de 0.70 recomendado por Nunnally y Bernstein (1994). La fiabilidad compuesta representa la varianza extraída entre el grupo de variables observadas y el constructo fundamental (Fornell y Larcker, I 98I). Por lo general, un índice de fiabilidad compuesta (IFC) superior a 0.60 es considerado deseable (Bagozzi y Yi, 1988); en la investigación, este valor es ampliamente superado. El índice de la varianza extraída (IVE) fue calculado para cada uno de los constructos, resultando un IVE superior a 0.50 (Fornell y Larcker, (98I); en nuestro trabajo, el 0.50 se supera en todos los factores.

Por lo que respecta a la evidencia de la validez discriminante, la medición se proporciona en dos formas que se pueden apreciar en el cuadro 3. Primero, con un intervalo de $95 \%$ de confidencialidad, ninguno de los elementos individuales 
Cuadro 2. Consistencia interna y validez convergente del modelo teórico

\begin{tabular}{|c|c|c|c|c|c|c|c|}
\hline Variable & Indicador & $\begin{array}{c}\text { Carga } \\
\text { factorial }\end{array}$ & $\begin{array}{l}\text { Valor-t } \\
\text { Robusto }\end{array}$ & $\begin{array}{l}\text { Promedio } \\
\text { de la carga } \\
\text { factorial }\end{array}$ & $\begin{array}{l}\text { "a" de } \\
\text { Cronbach }\end{array}$ & IFC & IVE \\
\hline Innovación & $\begin{array}{l}\text { IPI } \\
\text { IP2 } \\
\text { IP3 }\end{array}$ & $\begin{array}{l}0.879 * * * \\
0.921^{* * * *} \\
0.833^{* * * *}\end{array}$ & $\begin{array}{l}1.000^{*} \\
25.755 \\
22.723\end{array}$ & 0.877 & 0.907 & 0.911 & 0.771 \\
\hline $\begin{array}{l}\text { Reducción de } \\
\text { los costos de } \\
\text { las compras }\end{array}$ & $\begin{array}{l}\text { PPI } \\
\text { PP2 } \\
\text { PP3 }\end{array}$ & $\begin{array}{l}0.883^{* * * *} \\
0.917^{* * * *} \\
0.901^{* * * *}\end{array}$ & $\begin{array}{l}1.000 * \\
26.665 \\
26.833\end{array}$ & 0.901 & 0.927 & 0.927 & 0.811 \\
\hline $\begin{array}{l}\text { Desempeño } \\
\text { financiero }\end{array}$ & $\begin{array}{l}\text { FR1 } \\
\text { FR2 } \\
\text { FR3 }\end{array}$ & $\begin{array}{l}0.893^{* * * *} \\
0.945^{* * * *} \\
0.921^{* * * *}\end{array}$ & $\begin{array}{l}1.000 * \\
29.412 \\
29.678\end{array}$ & 0.919 & $0.94 I$ & 0.943 & 0.846 \\
\hline $\begin{array}{l}\text { Colabora- } \\
\text { ción con los } \\
\text { proveedores }\end{array}$ & $\begin{array}{l}\text { CRI } \\
\text { CR2 } \\
\text { CR3 } \\
\text { CR4 }\end{array}$ & $\begin{array}{l}0.766^{* * *} \\
0.864^{* * *} \\
0.846 * * * \\
0.869^{* * * *}\end{array}$ & $\begin{array}{l}1.000 * \\
17.332 \\
16.001 \\
19.557\end{array}$ & 0.836 & 0.899 & 0.903 & 0.701 \\
\hline & & & $\begin{array}{l}<0.000) ; \\
\text { RMSEA = }\end{array}$ & .972 & ; CF & & \\
\hline
\end{tabular}

* = Parámetros costreñidos a ese valor en el proceso de identificación $* * *=p<0.001$

de los factores latentes de la matriz de correlación contiene el valor I.0 (Anderson y Gerbing, 1988). Segundo, la varianza extraída entre cada par de constructos es superior que su correspondiente IVE (Fornell y Larcker, I98I). Con base en estos criterios, puede concluirse que las distintas mediciones realizadas en este estudio demuestran suficiente evidencia de fiabilidad y validez convergente $y$ discriminante.

\section{Resultados}

El modelo conceptual propuesto en este estudio (figura I) se examinó utilizando el modelo de ecuaciones estructurales (SEM) con el software EQS 6.I (Bentler, 2005; Byrne, 2006; Brown, 2006). La estimación empírica de los principales 
efectos del modelo presentados en el cuadro 3 indican que el ajuste de los datos del modelo conceptual es aceptable $\left(S-B X^{2}=125.3213 ; \mathrm{df}=59 ; p=\right.$ $0.000 ; \mathrm{NFI}=0.972 ; \mathrm{NNFI}=0.98 \mathrm{I} ; \mathrm{CFI}=0.985 ; \mathrm{y}$ RMSEA $=0.059$ ). Para obtener los resultados estadísticos de las hipótesis de investigación se realizó un SEM con las mismas variables para comprobar la estructura del modelo y lograr los resultados que permitieran contrastar las hipótesis planteadas. La validez nomológica del modelo teórico fue analizada mediante el desempeño del test de la Chi Cuadrada, en el cual el modelo teórico fue comparado con la medición del modelo. Los resultados indican que las diferencias no significativas del modelo teórico son buenas en la explicación de las relaciones observadas entre los constructos latentes (Anderson y Gerbing, 1988; Hatcher, 1994). Los resultados se presentan en el cuadro 4.

Con respecto a la primera hipótesis $\mathrm{HI}$ en el cuadro 4 se pueden apreciar los resultados obtenidos $\beta=0.727, p<0.00$ I, lo cual indica que la colaboración con los proveedores tiene efectos significativos en la innovación. En cuanto a la segunda hipótesis $\mathrm{H} 2, \beta=0.016$, no significativa, señala que la colaboración con los proveedores no tiene repercusiones significativas en la reducción de los costos de las compras. Por último, en la tercera hipótesis $\mathrm{H} 3$, cuyos resultados fueron $\beta=0.22 \mathrm{I}, p<0.00 \mathrm{I}$, puede observarse que la colaboración con los proveedores tiene efectos importantes en el desempeño financiero. En

Cuadro 3.Validez discriminante de la medición del modelo teórico

\begin{tabular}{|c|c|c|c|c|}
\hline Variables & I & 2 & 3 & 4 \\
\hline I. Innovación & 0.771 & 0.697 & 0.346 & 0.712 \\
\hline $\begin{array}{l}\text { 2. Reducción de los } \\
\text { costos de las } \\
\text { compras }\end{array}$ & $0.693 / 0.977$ & $0.8 \mathrm{II}$ & 0.568 & 0.494 \\
\hline $\begin{array}{l}\text { 3. Desempeño } \\
\text { financiero }\end{array}$ & $0.452 / 0.704$ & $0.634 / 0.874$ & 0.846 & 0.342 \\
\hline $\begin{array}{l}\text { 4. Colaboración con } \\
\text { los proovedores }\end{array}$ & $0.694 / 0.994$ & $0.569 / 0.837$ & $0.457 / 0.713$ & 0.701 \\
\hline
\end{tabular}

La diagonal representa el índice de la varianza extraída (IVE), mientras que por encima de la diagonal se presenta la parte de la varianza (la correlación al cuadrado). Por debajo de la diagonal, se presenta la estimación de la correlación de los factores con un intervalo de confianza de $95 \%$. 
Cuadro 4. Resultados del sistema de ecuaciones estructurales DEL MODELO DE COLABORACIÓN

\begin{tabular}{lcccc}
\hline Hipótesis & $\begin{array}{c}\text { Relación } \\
\text { estructural }\end{array}$ & $\begin{array}{c}\text { Coeficiente } \\
\text { estandarizado }\end{array}$ & Valor $\mathrm{t}$ & $\begin{array}{c}\text { Medida } \\
\text { de los FIT }\end{array}$ \\
\hline $\begin{array}{l}\text { HI.A mayor co- } \\
\text { laboración con los } \\
\begin{array}{l}\text { proveedores, mayor } \\
\text { nivel de innovación }\end{array}\end{array}$ & $\begin{array}{c}\text { Colaboración } \\
\text { Innovación }\end{array}$ & $0.727^{* * *}$ & 8.638 & $\mathrm{x}_{(59)}^{2}=\begin{array}{l}|25.32| 3 \\
\mathrm{P}=0.000\end{array}$ \\
\end{tabular}

\begin{tabular}{|c|c|c|c|c|}
\hline $\begin{array}{l}\text { H2.A mayor } \\
\text { Colaboración con } \\
\text { los proveedores, } \\
\text { menores costos de } \\
\text { las compras }\end{array}$ & $\begin{array}{c}\text { Colaboración } \\
\text { Reducción de } \\
\text { costos }\end{array}$ & 0.016 & 0.147 & $\begin{aligned} \mathrm{NFI} & =0.972 \\
\mathrm{NNFI} & =0.98 \mathrm{I}\end{aligned}$ \\
\hline $\begin{array}{l}\text { H3.A mayor } \\
\text { colaboración con los } \\
\text { proveedores } \\
\text { mayor desempeño } \\
\text { financiero }\end{array}$ & $\begin{array}{l}\text { Colaboración } \\
\qquad \\
\text { Desempeño } \\
\text { financiero }\end{array}$ & $0.221 * * *$ & 2.821 & $\begin{aligned} \mathrm{CFI} & =0.985 \\
\mathrm{RMSEA} & =0.059\end{aligned}$ \\
\hline
\end{tabular}

$* * *=p<0.001$.

resumen, se comprueba que, de las tres variables desde las cuales puede medirse la colaboración con los proveedores, los niveles de innovación y de desempeño financiero tienen un buen efecto significativo $(p<0.00 \mathrm{I})$.

\section{Discusión}

Si consideramos que innovar es detectar una posible mejora en un producto, servicio o proceso ejecutable, no podemos afirmar que las empresas productoras de muebles con un alto nivel de colaboración con sus proveedores sean las más innovadoras, sino que están en mejores condiciones de gestionar los pasos necesarios para la innovación. Además, no debe olvidarse que se está analizando un sector tradicional, el de la industria del mueble, que se ha desarrollado 
mediante la transformación de los talleres artesanales, principalmente dirigidos a satisfacer una demanda local, en fábricas adaptadas a mercados mucho más amplios. Una consecuencia de este proceso de desarrollo es el elevado grado de concentración espacial de la industria de España, que se localiza sobre todo en la Comunidad Valenciana y en la Comunidad de Cataluña (Camisón, Roig y Torcal, 1992).

En este sentido, a los empresarios del sector del mueble les cuesta demasiado ejecutar los cambios necesarios para desarrollar las actividades de innovación. En primer lugar, porque las empresas requieren flexibilidad para tomar decisiones, y por lo general los empresarios y empleados no siempre asumen su papel de impulsores de la innovación. En segundo lugar, porque para efectuar un cambio en las actividades de innovación hace falta preparación y conocimiento, es decir, si no se conoce la forma de actuar de los clientes potenciales y de las empresas que participan en el proceso de colaboración es difícil orientar el cambio y, por supuesto, llevarlo a cabo. Finalmente, la actual crisis productiva que enfrenta el sector del mueble de España y el incremento de las importaciones están obligando a los empresarios a reestructurar y reorganizar sus negocios. Sin lugar a dudas, ante la fuerte competencia internacional del mueble en un mercado cada vez más global, el crecimiento del sector pasa por la diferenciación (ANIEME, 2006).

China es el principal proveedor de muebles extranjeros en el mercado español $y$, a pesar de la baja calidad de sus productos, compite con costos muy bajos, lo que redunda en que se compren más muebles chinos que españoles en el mercado interno. Lo anterior es preocupante porque, en un entorno tradicional que crece por encima de $4 \%$, las empresas productoras de muebles tienen verdaderos problemas para vender sus productos, tanto dentro como fuera de sus fronteras (ANIEME, 2006). Esto permite ver con claridad la problemática actual del sector: sus costos no son competitivos comparados con los de otros países, sus productos no se diferencian de los importados y, además, carecen de una marca propia. Por lo tanto, el principal reto de los empresarios del sector del mueble en España es implantar la colaboración con los proveedores en un afán de buscar la reducción de los costos y aprovechar los avances tecnológicos como una estrategia -interna y externa-, de crecimiento, que les permita aprovechar las oportunidades que brindan los mercados. 
Si bien durante 2006 las empresas del mueble de España alcanzaron ventas por un poco más de mil millones de euros, el país registró en el mismo período importaciones de muebles cercanas a dos mil millones de euros (ANIEME, 2006), arrojando un déficit de cerca de mil millones de euros en la balanza comercial. No obstante estas cifras, existen varias empresas del ramo, sobre todo algunas medianas y la mayoría de las grandes, que obtuvieron beneficios adicionales en el lapso analizado. La clave está, según la ANIEME (2006), en buscar un nuevo modelo de negocio basado en la innovación y orientado al consumidor, para garantizar un mejor desempeño financiero. Así, las antiguas fábricas han ido dando paso a empresas modernas, donde cerca de $10 \%$ de los recursos se dedican a innovar, gestionar y vender los productos. Junto a estos elementos, se subraya la necesidad de una colaboración más estrecha con los proveedores y con otros sectores relacionados con el hábitat, como el textil, el de la iluminación, el de la cerámica y el inmobiliario. La máxima expresión de tal colaboración sería en contract, la venta en paquete de los productos de las distintas industrias, para lograr un mayor desempeño financiero de las empresas participantes.

Así, los resultados de esta investigación demuestran claramente que la colaboración con los proveedores es benéfica para todas las empresas involucradas de la industria del mueble de España. Por lo tanto, resulta ilustrativo el hecho de que hoy en día pueda iniciarse una estrecha colaboración entre empresas y proveedores, y que las repercusiones sean positivas para todos, sin que esto signifique perder el control de los canales de distribución por parte de las empresas productoras de muebles. Cabe agregar que estos resultados no eran muy obvios unos cuantos años atrás.

Implicaciones para los gestores

La colaboración con los proveedores no necesariamente garantizará un buen desempeño de las organizaciones empresariales participantes, y no siempre tendrá un efecto positivo en los negocios, pero sí permite a las empresas implicadas en el proceso de colaboración ejecutar de manera conjunta una serie de acciones que les hagan posible alcanzar sus metas. En este sentido, la colaboración con los proveedores requiere buenos mecanismos de control y gestión en los cuales se involucren todos los negocios participantes, para que el efecto en el desempeño de estos últimos sea positivo. Es por ello que en el proceso de colaboración tanto las empresas como los proveedores de 
servicios deben obtener beneficios mutuos, ya que de eso dependerán las futuras relaciones de colaboración y el eficiente funcionamiento de los negocios.

Es precisamente este aspecto lo que permitirá que las organizaciones empresariales se esfuercen no sólo en lograr un mejor desempeño particular, sino también uno mejor de todas las organizaciones involucradas, tomando en consideración, entre otros aspectos, la innovación, la reducción de los costos de las compras y el desempeño financiero. Asimismo, los gestores requieren un mayor involucramiento en la toma de decisiones, ya que aquellas que se tomen afectarán a todas las organizaciones empresariales participantes. Por ello, los gestores deben buscar nuevas formas de obtener una mejor actuación en los negocios mediante las distintas actividades que genera la colaboración. Además, cada sector empresarial tiene diferentes grados de colaboración y cooperación con sus proveedores, lo que origina variadas repercusiones en su desempeño. Quizá hoy en día el sector del mueble en España tenga un gran potencial en el desarrollo y los beneficios que entraña la colaboración.

\section{Limitaciones y estudios futuros}

Este estudio se basa en una muestra que por su tamaño representa una limitación, aunque ésta se subsana por la calidad de los encuestados y por la experiencia en el área de la logística. Con todo, las diferencias en la implantación de la colaboración con los proveedores entre las empresas del sector del mueble en España pueden influenciar los resultados logrados en su desempeño. Otro inconveniente es la imposibilidad de obtener toda la información necesaria, puesto que se ha extraído solamente una parte de la información respecto de las relaciones de la colaboración con los proveedores, pues la mayoría de las empresas de la muestra considera la información requerida altamente confidencial y privada, por lo cual los datos a los que se tuvo acceso no necesariamente reflejan el desempeño real de las empresas. A pesar de ello, otros elementos de medición del desempeño real, por ejemplo los precios, pueden ser adecuados para incrementar la validez de los resultados en futuros estudios. Es esencial ir más allá de los resultados técnicos y discutir qué tipo de empresas proveedoras de servicios realiza una mejor colaboración con las empresas productoras de muebles, y qué actividades son más importantes en la relación de colaboración. Preguntas que se pueden responder en trabajos posteriores. 


\section{FUENTES CONSULTADAS}

Adobor, H. (2006). "Inter-firm collaboration: configurations and dynamics". Competitiveness Review, 16 (2), I22-134.

Anderson, J.C. y D. Gerbing ( 1988 ). "Structural equation modeling in practice: a review and recommended two-step approach". Psychological Bulletin, |3, 4| |-423.

y J.A. Narus (1990). "A model of distributor firm and manufacturer firm working partnerships”. Journal of Marketing, 54 (I), 42-58.

ANIEME (2006). Informe enero-septiembre 2006 del comercio exterior del mueble.

Documento de trabajo.Valencia:Asociación Nacional de Industriales y Exportadores de Muebles de España.

Bagozzi, R. y Y.Yi (1988). "On the evaluation of structural equation models". Journal of the Academy of Marketing Science, 16 (I), 74-94.

Bentler, P.M.(1990).“Comparative fit indexes in structural models”. Psychological Bulletin, 107 (2), 238-246.

(2005). EQS 6 Structural Equations Program Manual [en línea]. Encino, Multivariate Software. Disponible en: www.mvsoft.com

y D. Bonnet (1980). "Significance tests and goodness of fit in analysis of covariance structures”. Psychological Bulletin, 88, 588-606.

Bititci, U.S. et al. (2004). “Creating and managing value in collaborative networks”. International Journal of Physical Distribution \& Logistics Management, 34 (3-4), 25I-268.

Bowersox, D.J., D.J. Closs y T.P. Stank (2003). “How to master cross-enterprise collaboration”. Supply Chain Management Review, 7 (4), I 8-27.

Brown, T. (2006). Confirmatory Factor Analysis for Applied Research. Nueva York: The Guilford Press.

Byrne, B.M. (1989). A Primer of LISREL: Basic Applications and Programming for Confirmatory Factor Analysis Analytic Models. Nueva York: Springer.

(2006). Structural Equation Modeling with EQS, Basic Concepts,Applications, and Programming. $2^{\mathrm{a}}$ ed. Londres: LEA Publishers.

Camisón, C., S. Roig y R. Torcal ( 1992). Análisis sectorial del mueble y la madera, Collecció Monografies de l'Horta-Sud núm. 3, España.

Cannon, J.P.y C. Homburg (200I). "Buyer-supplier relationships and customer firm costs”. Journal of Marketing, 65 (I), 29-43. 
Chau, P.(I997)." Reexamining a model for evaluating information center success using a structural equation modeling approach”. Decision Sciences, 28 (2), 309-334.

Chou, C.P., P.M. Bentler y A. Satorra (199I). "Scaled test statistics and robust standard errors for non-normal data in covariance structure analysis". British Journal of Mathematical and Statistical Psychology, 44, 347-357.

Corbett, C.J., J.D. Blackburn y L.N.Van Wassenhove (I999). "Partnerships to improve supply chains". Sloan Management Review, 40 (4), 7I-82.

Corsten, D.y J. Felde (2005). "Exploring the performance effects of key-supplier collaboration". International Journal of Physical Distribution \& Logistics Management, 35 (6), 445-46I.

Cross, R., S.P. Borgatti y A. Parker (2002). "Making invisible work visible”. California Management Review, 44 (2), 25-46.

Dröge, C.y R. Germain (2000)."The relationship of electronic data interchange with inventory and financial performance". Journal of Business Logistics, 2I (2), 209-230.

Dyer,J.H.y H.Singh (1998).“The relational view: cooperative strategy and sources of inter-organizational competitive advantage". Academy of Management Review, 23 (4), 660-679.

Ellram, L.M. (1995). "Total cost of ownership”. International Journal of Physical Distribution \& Logistics Management, 25 (8), 4-23.

- y O.R.V.Edis ( 1996). "A case study of successful partnering implementation". International Journal of Purchasing and Materials Management, $32(2), 20-28$.

Fisher, M.L. (1997). "What is the right supply chain for your product?". Harvard Business Review, 75 (2), I05-I I6.

Fornell, C. y D. Larcker (I98I). "Evaluating structural equation models with unobservable variables and measurement error".Journal of Marketing Research, 18, 39-50.

Gadde, L.E., L. Humer y H. Hakansson (2003). "Strategizing in industrial networks". Industrial Marketing Management, 32 (5), 357-364.

Gilley, K.M. y A. Rasheed (2000). "Making more by doing less: an analysis of outsourcing and its effects on firm performance". Journal of Management, 26 (4), 763-790.

Golicic, S.L., J.H. Foggin y J.T. Mentzer (2003). “Relationship magnitude and its role in inter-organizational relationship structure".Journal of Business Logistics, 24 (I), 57-75. 
Hair, J.F. et al. (1995). Multivariate Data Analysis with Readings. Nueva York: Prentice-Hall.

Hardy, C., N. Phillips y T.B. Lawrence (2003).“"Resources, knowledge and influence: the organizational effects of inter-organizational collaboration".Journal of Management Studies, 40 (2), 32 I-347.

Hatcher, L. (1994). A Step by Step Approach to Using the SAS System for Factor Analysis and Structural Equation Modeling, Cary: SAS Institute Inc.

Heck, R.H. (1998).“Factor analysis: exploratory and confirmatory approaches”, en G.A. Marcoulides (ed.). Modern Methods for Business Research. Mahwah: Lawrence Erlbaum Associates, 177-2I 5.

Heide,J.B.y G.John (1990)."Alliances in industrial purchasing: the determinants of joint action in buyer-supplier relationships". Journal of Marketing Research, 27 (I), 24-36.

Holweg, M. et al.(2005)."Supply chain collaboration:making sense of the strategic continuum”. European Management Journal, 23 (2), I70-I8I.

Hu, L.T., P.M. Bentler y Y. Kano (1992). "Can test statistics in covariance structure analysis be trusted?". Psychological Bulletin, II2, 35I-362.

Huxham, C. (1996). Creating Collaborative Advantage. Londres: Sage Publications.

Inkpen, A.C. (1996). “Creating knowledge through collaboration”. California Management Review, 39 (I), I23-I40.

Jap, S.D. (1999)." "Pie-expansion efforts: collaboration processes in buyer-supplier relationships". Journal of Marketing Research, 36 (4), 46I-475.

(200I). "Pie sharing' in complex collaboration context". Journal of Marketing Research, 38 (I), 86-99.

Jordan, J.A. y F.J. Michel (2000). Next Generation Manufacturing: Methods and Techniques. Chichester: John Wiley and Sons.

Jorde, T.M. y D.J. Teece (1990). “Innovation and cooperation: implications for competition and antitrust”. Journal of Economic Perspectives, 4 (3), 75-96.

Jöreskog, K.G. y D. Sörbom (1986). LISREL VI: Analysis of Linear Structural Relationships by Maximum Likelihood, Instrumental Variables and Square Methods. Mooresville: Scientific Software.

Konczak, L.J. (200I). "The process of business/environmental collaborations: partnering for sustainability”. Personnel Psychology, 54 (2), 5I 5-5 I 8. 
Lambert, D.M. y M.C. Cooper, (2000), "Issues in supply chain management”, Industrial Marketing Management, 29 (I), 65-83. , A.M. Knemeyer y J.T. Gardner (2004). "Supply chain partnerships: model validation and implementation". Journal of Business Logistics, 25 (2), 2 I-42.

—, M.A. Emmelhainz y J.T. Gardner (1999). "Building successful partnerships”. Journal of Business Logistics, 20 (I), I65-I8I.

Lee, H.L., V. Padmanabhan y S. Whang (1997). "The bullwhip effect in supply chains”. Sloan Management Review, 38 (3), 93-102.

Mentzer, J.T., J.H. Foggin y S.L. Golicic (2000). "Collaboration: the enablers, impediments and benefits". Supply Chain Management Review, 5, 52-58.

et al. (200I). "What is supply chain management?", en J.T. Mentzer (ed.). Supply Chain Management. Thousand Oaks: Sage.

Min, S. et al. (2005). "Supply chain collaboration: what's happening?" The International Journal of Logistics Management, 16 (2), 237-256.

Nunnally, J.C. e I.H. Bernstein (1994). Psychometric Theory. $3^{\text {a }}$ ed. Nueva York: McGraw-Hill.

Papke-Shields, K.E., M.J. Malhotra y V. Grover (2002). "Strategic manufacturing planning systems and their linkage to planning system success”. Decision Science, I 3 ( I), I-30.

Perks, H. (200I). “Marketing information exchange mechanisms in collaborative new product development”. Industrial Marketing Management, 29 (2), 179-189.

Powell,W.W.(1998).“Learning from collaboration: knowledge networks in the biotechnology and pharmaceutical industries". California Management Review, 40 (3), 228-240.

et al. (2005). "Network dynamics and field evolution: the growth of inter-organizational collaboration in the life sciences". The American Journal of Sociology, I 10 (4), I I 32- I 206.

Quinn, F.J. (1999). "Cooperation and collaboration: the keys to supply chains success”. Logistics Management \& Distribution, 38 (2), 35-40.

Sabath, R.E. y J. Fontanella (2002). "The unfulfilled promise of supply chain collaboration”. Supply Chain Management Review, 6 (4), 24-29.

Satorra, A. y P.M. Bentler (1988). "Scaling corrections for chi square statistics in covariance structure analysis". American Statistics Association 1988 Proceedings of the Business and Economic Sections, 208-3I 3. 
Sawhney, M. (2002). “Don't just relate-collaborate”. MIT Sloan Management Review, 43 (3), 96-97.

Scannell, T.V., S.K. Vickery y C.L. Dröge (2000). "Upstream supply chain management and competitive performance in the automotive supply industry". Journal of Business Logistics, 2 I (I), 23-48.

Segars,A.H.yV. Grover (1993)."Re-examining perceived ease of use and usefulness: a confirmatory factor analysis". MIS Quarterly, I7 (4), 5I 7-525.

Simatupang, T.M. y R. Sridharan (2002). "The collaborative supply chain". The International Journal of Logistics Management, I 3 (I), I5-30.

(2005a), “An integrative framework for supply chain collaboration”. The International Journal of Logistics Management, 16 (2), 257-274.

(2005b), "Supply chain discontent". Business Process Management Journal, I I (4), 349-369.

,A.C.Wright y R. Sridharan (2002). "The knowledge of coordination for supply chain integration”. Business Process Management Journal, 8 (3), 289-308.

Simonin, B.L. (1997).“The importance of collaborative know-how: an empirical test of the learning organization". Academy of Management Journal, 40 (2), II I50-II 74.

Singh, K.yW. Mitchell (1996).“Precarious collaboration: business survival after partners shut down or from new partnerships". Strategic Management Journal, I7 (7), 99-II5.

Spekman, R.E., D.J. Salmond y C.J. Lambe (1997)."Consensus and collaboration: norm-regulated behavior in industrial marketing relationships". European Journal of Marketing, 3 I (I I/I2), 832-856.

Sriram, V., R. Krapfel y R.E. Spekman (1992). “Antecedents to buyer-seller collaborations: an analysis from the buyer's perspective". Journal of Business Research, 25 (4), 303-320.

Stank, T.P., B.S. Keller y P.J. Daugherty (200I). "Supply chain collaboration and logistical service performance". Journal of Business Logistics, 22 (I), 29-48. ,P.J.Daugherty y C.W.Autry (1999)."Collaborative planning: supporting automatic replenishment programs". Supply Chain Management: An International Journal, 4 (2), 75-85.

Stefansson, G. (2006). "Collaborative logistics management and the role of the third-party service providers". International Journal of Physical Distribution \& Logistics Management, 32 (2), 76-92. 
Stern, A.J. y T. Hicks (2000). The Process of Business/Environmental Collaborations: Partnering for Sustainability. Westport: Quorum.

Stern, L.W. y T. Reeve (1980). "Distribution channels as political economies: a framework for comparative analysis". Journal of Marketing, 44 (3), 52-64.

Thron, T., G. Nagy y N. Wassan (2006). "The impact of various levels of collaborative engagement on global and individual supply chain performance". International Journal of Physical Distribution \& Logistics Management, 36 (8), 596-620.

Tracey, M.A., M.A. Vonderembse y J.S. Lim (1999). “Manufacturing technology and strategy formulation: keys to enhancing competitiveness and improving performance". Journal of Operations Management, I4 (4), $4 \mid \mathrm{I}-428$.

Tuominen, M. (2004). “Channel collaboration and firm value proposition”. International Journal of Retail \& Distribution Management, 32 (4), I78-I89.

Vereecke, A. y S. Muylle (2006). "Performance improvement through supply chain collaborative in Europe". International Journal of Operations \& Production Management, 26 (I I), I I 76- I I 98.

Von Hippel, E. (1988). The Sources of Innovation. Nueva York: Oxford University Press.

Walker, B., D. Bovet y J. Martha (2000). "Unlocking the supply chain to build competitive advantage". The International Journal of Logistics Management, II (2), I-8.

Walter, A. et al. (2003). "Functions of industrial supplier relationship and their impact on relationship quality". Industrial Marketing Management, 32, I59-169.

Wilding, R. y A. Humphries (2006). “Understanding collaborative supply chain relationships through the application of theWilliamson organizational failure framework". International Journal of Physical Distribution \& Logistics Management, 36 (4), 309-329.

Williamson, O.E. (1985). The Economic Institutions of Capitalism, Nueva York: The Free Press.

Zaheer, A., B. McEvily y V. Perrone (1998). “Does trust matter? Exploring the effects of inter-organizational and interpersonal trust on performance”. Organization Science, 9 (2), I4 I-I 59. 